\title{
Polymicrobial Injection-Site Abscesses Associated with Contaminated Methylprednisolone Injections in Florida
}

\author{
James Matthias*, Philip Cavicchia, Scott Pritchard and Robert Bernstein \\ Florida Department of Health, Tallahassee, Florida, USA
}

*Corresponding author: James Matthias, Florida Department of Health, Tallahassee, USA

Submission: 㘹August 31, 2017; Published: 㘹 November 02, 2017

\begin{abstract}
Objective: Identify injection-site abscesses and quantify riskfactors frominjections with subsequently recalled preservative-free Methylprednisolone acetate (pf-MPA) from Main Street Family Pharmacy (MSFP).

Design: Outbreak investigation and retrospective cohort study.

Setting: Private primary healthcare facility.

Patients: Three hundred and seven patients that received pf-MPA injections manufactured by MSFP from December 1, 2012 through February 28 2013.

Methods: Medical records were abstracted for individuals with an adverse event and individuals with known exposure to pf-MPA lots 011413dan and 120612dan. Records were analyzed for factors related to injection-site adverse events using logistic regression. Facility records from 2006 through 2013 were queried for International Classification of Diseases, $9^{\text {th }}$ edition, Clinical Modification (ICD-9-CM) diagnosis codes 682.5 and 680.5 to identify additional adverse events.

Results: Sixteen of 307 injected individuals (attack rate $=5.2 \%$ ) were identified with adverse events consisting of soft tissue infections at the injection site. Increasing body mass index (BMI) (odds ratio $[\mathrm{OR}]=1.17$; $95 \% \mathrm{CI}=1.04-1.33$ ), age of vial at injection (OR=1.13; 95\% CI=1.00-1.27), and date of injection $(\mathrm{OR}=10.01 ; 95 \% \mathrm{CI}=1.49-67.11)$ were associated with adverse events.

Conclusion: Risk of soft tissue infections following injection of recalled MSFP pf-MPA was greater among persons with higher BMI, receiving older product, and receiving injections from February 5 through February 15, 2013. Appropriate needle length was not used for patients with a greater BMI. A written protocol for pf-MPA injections including needle length and gauge should be created and trainings on this protocol provided to staff administering injections. Compounded pharmaceuticals, especially preservative-free formulations, should not be utilized for multiple injections.
\end{abstract}

\section{Introduction}

Intramuscular injections have been identified as the cause of many infectious disease outbreaks in the United States. Identified sources include intrinsically contaminated product or a breakdown in infection control practices leading to extrinsic contamination of the product [1-5] In September 2012, a national outbreak investigation involving the New England Compounding Center (NECC), identified at least 61 deaths and 749 adverse events from intrinsically contaminated preservative-free Methylprednisolone acetate (pf-MPA) primarily injected through epidural or intraspinal sites [6-9]. In addition to the NECC outbreak as of 2012, 11 outbreaks are known to have occurred since January 2000 in which compounded products have resulted in an additional 207 infections and 17 deaths [10]. On May 28, 2013, the Main Street Family Pharmacy (MSFP) of Tennessee recalled all lots of sterile compounded products manufactured since December 1, 2012 [11]. On June 7, 2013, the Food and Drug Administration (FDA) reported that it had identified bacterial and fungal growth from two separate lots of unopened $80 \mathrm{mg} / \mathrm{ml}, 10 \mathrm{~mL}$ vials of pf-MPA from MSFP; lot 011413dan and lot 010913dan [12]. In total, 96 facilities in 17 states received sterile compounded products from MSFP, including pf-MPA [12]. Three of these facilities were located in Florida; however, only one of the Florida facilities received the recalled pf-MPA $(80 \mathrm{mg} / \mathrm{ml}, 10 \mathrm{~mL}$ vials) and reported potential adverse events among injected patients. As of June 27, 2013, the Centers for Disease Control and Prevention (CDC) reported that 26 individuals in four states had an infection suspected to be associated with exposure to the recalled MSFP products [11]. The majority of these individuals had localized skin and soft tissue infections at the site of the injection, and all affected individuals had been exposed to a preservative-free formulation of MSFP MP It was determined that among the three Florida recipient facilities, one facility received recalled pf-MPA from MSFP and reported potential adverse events among injected patients. On May 28, the Florida Department of Health (DOH) sent a team to the facility to investigate. The aims 
of the investigation were to identify adverse events from pf-MPA injections from MSFP and to quantify any associated risk factors.

\section{Materials and Methods}

A case of injection-site abscess was defined as a person who developed a suspected infection associated with injection of a product labeled as sterile, that was distributed by MSFP in Newbern, Tennessee, and used at the facility from December 1, 2012 through February 28, 2013. After February 28, the facility stopped using all pf-MPA MSFP products. Case-patients were identified by facility staff and through review of medical records (including records from nearby facilities where patients sought treatment). Records of all patients receiving an injection with pfMPA from MSFP from December 1, 2012 through March 1, 2013 were reviewed to estimate lot-specific attack rates. Patients with recorded exposure to only lots 011413 dan and 120612dan were further examined to identify risk factors associated with infection. The six-digits in the lot number reference the date the product was manufactured. Abstracted patient data consisted of basic demographic information, weight, height, body mass index (BMI), co-morbidities, past medical history, social history, injection history, follow-up medical visits, chief complaints, discharge diagnoses, and any related laboratory results. A significant portion of individuals who experienced adverse events and who were identified prior to abstracting records had received an injection between February 5 and 15, 2013. Accordingly, a categorical variable for injection date was created for injections prior to February 5 from February 5 through February 15 and after February 15. Product age in days was determined by subtracting the date of injection from the date of manufacture for the product. In addition to the medical record abstraction of patient visits occurring from December 1, 2012 through February 28, 2013 the facility database was queried from July 2006 through July 2013 to identify additional adverse events and to determine the background rate of occurrence of abscesses using International Classification of Diseases, 9th edition, Clinical Modification (ICD- 9-CM) diagnosis codes. Diagnosis codes 682.5 (abscess of the buttocks) and 680.5 (carbuncle or furuncle of the buttocks) were used for the query as all previously identified adverse health events had one of these discharge diagnoses recorded. For univariate analysis, comparing cases to non-cases exposed to lots 011413dan or 120612dan, Student's T-test was used for continuous variables and the Mann-Whitney $U$ test was used for categorical variables. Logistic regression was conducted to identify risk factors for adverse events while controlling for demographic factors, comorbidities, and injection-related risk factors. For the logistic regression model, variables found to be statistically significant in the univariate analysis and variables that have been identified in the literature to be significant risk factors such as gender, age, vial age at injection, and underlying diabetes were included in the final model [1,8,13-15]. All data in this investigation were analyzed using Epi Info 7.1.2.0 (CDC, Atlanta, GA).

\section{Result}

Table 1: Characteristics of 16 Patients with Injection-Site Abscesses.

\begin{tabular}{|c|c|}
\hline Characteristics & $n=16$ \\
\hline Age (years), mean (range) & $44.4(23-70)$ \\
\hline \multicolumn{2}{|l|}{ Sex, No. (\%) } \\
\hline Male & $4(25)$ \\
\hline Female & $12(75)$ \\
\hline BMI $\left(\mathrm{kg} / \mathrm{m}^{2}\right)$, mean (range) & $34.1(26-55)$ \\
\hline \multicolumn{2}{|l|}{ Co-morbidities, No. (\%) } \\
\hline Hypertension & $7(44)$ \\
\hline Hyperlipidemia & $5(31)$ \\
\hline Heart disease & $4(25)$ \\
\hline Diabetes & $2(13)$ \\
\hline COPD & $1(6)$ \\
\hline Vial age at injection (days), median (range) & $25(14-77)$ \\
\hline Time from injection to diagnosis (days), median (range) & $36(19-115)$ \\
\hline \multicolumn{2}{|l|}{ Treatment, No. (\%) } \\
\hline Antibiotics prescribed & $15(94)^{*}$ \\
\hline Incision and drainage & $13(81)$ \\
\hline \multicolumn{2}{|l|}{ Microbiology results, No. (\%) } \\
\hline Positive & $6(38)$ \\
\hline Negative & $1(6)$ \\
\hline Not performed & $9(56)$ \\
\hline
\end{tabular}

The remaining patient's antibiotic status was unknown 
Table 2: Attack rates by recorded MSFP lot number.

\begin{tabular}{|c|c|c|c|}
\hline Lot Number & Cases & Exposed & Attack Rate \\
\hline 011413dan & 13 & 121 & $10.70 \%$ \\
\hline 120612dan & 2 & 28 & $7.10 \%$ \\
\hline 092712dan & 1 & 1 & $100 \%$ \\
\hline Other lots* & 0 & 57 & $0.00 \%$ \\
\hline Unknown lots & 0 & 100 & $0.00 \%$ \\
\hline Total & 16 & 307 & $5.20 \%$ \\
\hline
\end{tabular}

Included 092212dan, 100112dan, 113012dan, 121912dan, 010213dan, and 010913dan

Table 3: Comparison Between Cases and non-Cases with Recorded Exposure to Lots 011413dan or 120612dan of pf-MPA from MSFP.

\begin{tabular}{|c|c|c|c|}
\hline Characteristic & Cases (n=15) & non-Cases $(n=128)$ & P-value \\
\hline \multicolumn{4}{|l|}{ Age (years) } \\
\hline Mean (range) & $45.5(23-70)$ & $51.0(13-83)$ & 0.22 \\
\hline Sex, No. (\%) & & & 0.43 \\
\hline Male & $3(20)$ & $38(30)$ & \\
\hline Female & $12(80)$ & $90(70)$ & \\
\hline \multicolumn{4}{|l|}{ BMI $\left(\mathrm{kg} / \mathrm{m}^{2}\right)$} \\
\hline Mean (range) & $34.7(26-55)$ & $30.0(17-51)$ & 0.01 \\
\hline \multicolumn{4}{|l|}{ Co-morbidities, No. (\%) } \\
\hline Hypertension & $7(47)$ & $75(59)$ & 0.38 \\
\hline Hyperlipidemia & $5(33)$ & $55(43)$ & 0.47 \\
\hline Diabetes & $2(13)$ & $16(13)$ & 0.92 \\
\hline Heart disease & $4(27)$ & $28(22)$ & 0.67 \\
\hline COPD & $1(7)$ & $15(12)$ & 0.56 \\
\hline Injection date, No. (\%) & & & $<0.01$ \\
\hline Pre 2/5/13 & $4(27)$ & $88(69)$ & \\
\hline $2 / 5 / 13-2 / 15 / 13$ & $11(73)$ & $19(15)$ & \\
\hline Post 2/15/13 & $0(0)$ & $21(16)$ & \\
\hline \multicolumn{4}{|l|}{ Vial age at injection (days) } \\
\hline Median (range) & $23.5(14-32)$ & $19(1-47)$ & 0.04 \\
\hline
\end{tabular}

Table 4: Odds of Having an Adverse Event for Patients with Recorded Exposure to Lots 011413dan or 120612dan of PF-MPA from MSFP.

\begin{tabular}{|c|c|c|}
\hline Characteristic & Unadjusted OR $(95 \% \mathrm{CI})$ & Adjusted $\mathrm{OR}^{*}(95 \% \mathrm{CI})$ \\
\hline BMI $\left(\mathrm{kg} / \mathrm{m}^{2}\right)$ & $1.09(1.01,1.16)$ & $1.17(1.04,1.33)$ \\
\hline Age (years) & $0.98(0.95,1.01)$ & $0.95(0.90,1.01)$ \\
\hline \multicolumn{3}{|l|}{ Sex } \\
\hline Female & Reference & Reference \\
\hline Male & $0.59(0.16,2.22)$ & $0.28(0.04,1.98)$ \\
\hline \multicolumn{3}{|l|}{ Diabetes } \\
\hline No & Reference & Reference \\
\hline Yes & $1.08(0.22,5.22)$ & $0.23(0.02,3.21)$ \\
\hline \multicolumn{3}{|l|}{ Date of injection } \\
\hline Pre $2 / 5 / 13$ & Reference & Reference \\
\hline $2 / 5 / 13$ to $2 / 15 / 13$ & $12.74(3.66,44.33)$ & $10.01(1.49,67.11)$ \\
\hline Age of vial at injection (day) & $1.04(0.99,1.09)$ & $1.13(1.00,1.27)$ \\
\hline
\end{tabular}

Adjusted OR was calculated using a logistic regression model using all the variables present in this table 
MSFP pf-MPA was supplied to the facility in $10 \mathrm{ml}$ vials $(80 \mathrm{mg} /$ $\mathrm{ml}$ ), but lot numbers were not included on invoices. Facility practice was to inject $1 \mathrm{ml}$ in the buttocks region using 1-1.5 inch needles. Vial labels did not include any indication of whether the product was for "single use" or "multiple uses" but the facility routinely used them for multiple patients over the course of several days. A total of 307 patients received one or more injections with MSFP pf-MPA from December 1, 2012 through February 28, 2013. Sixteen (5.2\%) patients met the case definition for an injection- site abscess (Table 1). Among the cases, mean age was 44.4 years (range $=23-70$ ), $75 \%$ $(\mathrm{n}=12)$ were women and mean BMI was $34.1 \mathrm{~kg} / \mathrm{m} 2$ (range $=26-55)$. Indications for corticosteroid treatment included allergic rhinitis or upper respiratory tract infection $(n=13)$, urticaria $(n=1)$ and musculoskeletal complaints $(n=2)$. Patients presented with signs or symptoms of injection- site abscesses typically described as raised, swollen, warm, firm and tender to touch, and erythematous indurations of varying size. Diagnosis was a median of 36 days (range=19-115) following injection. Thirteen patients underwent incision and drainage of the lesion at least once. Fifteen cases were treated with antibiotics as outpatients; none were hospitalized or died. For those undergoing surgical incision and drainage, surgical notes indicated moderate to large amounts (up to $400 \mathrm{ml}$ ) of purulent or bloody fluids and cavities extending deep into subcutaneous tissues. Samples of abscess fluid or tissues were submitted from seven patients for culture or histopathology. Six samples had positive culture results (five exposed to lot 011413dan and one exposed to lot 120612dan). One patient with positive culture results, exposed to lot 011413dan, grew Enterobacter cloacae, Enterobacter aerogenes and Klebsiella pneumonia. Of the remaining four samples from patients exposed to lot 011413dan two had cloacae $E$ and K pneumonia isolated and two had only K pneumonia isolated. Aspergillum versicolor was cultured from one patient exposed to lot 120612dan. Fungal testing was completed only on one patient exposed to lot $011413 \mathrm{dan}$ and no fungal elements were observed. Lot number was recorded in patient records for 207 $(67.4 \%)$ of 307 injections administered during the study period; at least nine different lots of pf-MPA were used (Table 2), but only three lots were associated with infection: 092712dan ( $\mathrm{n}=1), 120612$ dan $(n=2)$, and 011413 dan $(n=13)$. One case had recorded exposure to lot 011413 dan, but received injection with the product on January 10,2013 , suggesting this recorded lot was incorrect. All other cases associated with lot 011413 dan were injected during the threeweek period January 28 through February 15, 2013. FDA recovered bacteria (Bacillus pumilus, Bacillus cereus/thuringiensis/mycoides, Roseomonas gilardii) and fungi (Acinetobacter ursingii, Alternaria SP, Cladosporium $s p$ ) from an unopened vial of pf-MPA lot 011413dan obtained from MSFP [11]. FDA did not test unopened vials from lots 092712dan and 120612dan. FDA also recovered bacteria (Bacillus licheniformis) and fungi (Penicillium $s p$ ) from an unopened vial of lot $010913 \mathrm{dan}$; this lot was used for at least 13 injections at the facility but no injection-site abscesses were detected. Risk factors evaluated for developing an injection-site abscess among recipients of lots 120612 dan and 011413 dan included patient age, gender, diabetes status, injection date, and vial age for the 15 cases and 128 non-cases (Table 3). A multivariable logistic-regression model was developed to examine these identified associations while controlling for other risk factors (Table 4). Factors that were significantly associated with injection-site abscesses included increasing BMI (odds ratio=1.17; 95\% CI 1.04-1.33), increasing age of vial contents on day of injection (odds ratio=1.13; $95 \% \mathrm{CI}$ 1.00-1.27) and an eleven day injection period from February 5 through February 15, 2013 (odds ratio=10.01; 95\% CI 1.49-67.11). Age, sex, and diabetes status were included in the model but not significantly associated with an adverse event. Fifteen (94\%) of 16 cases received a discharge diagnosis of an abscess or carbuncle on the buttocks (ICD-9-CM diagnosis codes 682.5 or 680.5). In order to assess background rates of these codes, review of electronic medical records from July 2006 through November 2012 identified 80 patients with ICD-9-CM diagnosis codes of 682.5 or 680.5 . Only two of these patients had also received an antecedent injection of pf-MPA. One patient received a pf-MPA injection in 2008 and was diagnosed with an abscess 51 days later. However, at that time, the source of pf-MPA was not MSFP and medical records did not specifically indicate that the infection was located at the injection site. A second patient, who received an injection of MSFP pf-MPA in February 2012 was diagnosed with an injection-site abscess 33 days later. The abscess required surgical debridement; tissues were tested at CDC and found to contain bacteria (Bacteroides urealyticus, Staphylococcus epidermidis) and fungi (Rhizopus oryzae).

\section{Discussion}

This investigation identified 16 patients with adverse events associated with a preservative-free formulation of MPA compounded by MSFP. Thirteen of these individuals (76\%) had recorded exposure to a specific lot of pf-MPA that was demonstrated to be intrinsically contaminated. Access to complete medical records of most patients including lot numbers for the administered MPA allowed for detailed risk factor analysis. This analysis identified BMI, vial age at injection, and an 11 day injection period as risk factors for the development of adverse events following injection with pf-MPA from MSFP. Given the potential health risks associated with the injection of preservative-free compounded pharmaceutical products, it is imperative to identify risk factors associated with adverse events in order to implement effective preventative measures $[2,5,9,10]$. Remote electronic access to the complete medical records for the 143 patients with identified exposure to pf-MPA from MSFP lots numbered 011413dan or 1201612 dan greatly assisted in this investigation. However, lot number was not recorded for 114 of the 307 patients identified. Missing lot numbers appeared to be systematic and did not differ by lot; for one case, the lot number for pf-MPA associated with an injection-site abscess was retrospectively added to the medical record following identification of the adverse event. The majority of patients received pf-MPA injections for treatment of allergic rhinitis or upper respiratory infections. DEPO-MEDROL, the brand name of FDA-approved MPA does have an indicated use for "control of severe and incapacitating allergic conditions intractable to adequate trials of conventional treatment [16]". Treatment of these conditions with glucose corticoids is not recommended as first lines of treatment and adverse events of localized tissue atrophy have 
been identified with these types of injections $[17,18]$. Physicians need to be aware whether the product they are utilizing has been compounded. Compounded pharmaceuticals are to be considered single dose vials, but in this setting were used as multiple dose vials for multiple patients. Injection practices using multiple dose vials have been linked to at least 19 outbreaks and should only be utilized when absolutely necessary [1-5].There is a need for clear labeling of the intended use for compounded products, especially for preservative-free formulations as there is an increased risk of infection when used inappropriately [7]. In addition, given the inherent risk for microbial contamination of preservative-free compounded products compared to the FDA-approved products (containing preservatives), clinicians need to assess the benefit to the patient prior to utilizing these preservative-free products $[2,4,8-10,19]$. Increasing body fat, as measured by BMI in this investigation, was significantly associated with developing an abscess following injection of pf-MPA. Previous studies have identified the need to take into account weight and fat pad depths when administering intramuscular injection, and as such, alter needle length and gauge accordingly [20-22]. Although this facility did increase needle length (from 1 inch to 1.5 inches) for patients with BMI greater than 25 , the needle length selection was still likely insufficient to reach the intramuscular depth required for injection [22]. Facilities administering these types of injections should have written protocols for intramuscular injections that account for the increased fat pad of some patients and specify the appropriate needle length and gauge. Staff administering these injections should be trained and their performance should be audited on a regular basis. In a non-random sample of unopened vials of pf-MPA produced by MSFP, the FDA isolated B. pumilus $B$, cereus/thuringiensis/mycoides, gilardii $R$, ursingii A, Alternaria $s p$, Cladosporium SP, licheniformis B and Pencillium SP [12]. Although these microbes differ from the pathogens identified in this patient population it does not conclusively determine whether the adverse events were caused by wholly intrinsic or wholly extrinsic contamination of the product. However, medical records from as far back as July 2006 through December 2012 indicate pf-MPA intramuscular injections were administered regularly and only two individuals experienced similar adverse events during this timeframe. This suggests intrinsic contamination as the most likely source of these adverse events. Similar to the recent fungal meningitis outbreak caused by intrinsically contaminated pfMPA, the age of the vial at injection was a significant risk factor for developing an adverse event [8]. Given that the FDA found both bacterial and fungal contaminants in unopened vials of pfMPA from MSFP, it is not surprising that increasing vial age would be a risk factor for developing infection in this outbreak as well $[11,12]$. The FDA reported 25 separate observations during their on-site investigation of MSFP [23]. Some of the observed practices, including leaving vials opened to the environment for multiple hours, could have led to sporadic polymicrobial contamination of the product. The fact that nearly two-thirds of all adverse events clustered within an 11-day period, despite the product being injected for more than seven weeks, raises the question of whether sporadic intrinsic contamination of a few vials at MSFP might explain the increased attack rate during this period. Moreover, no noted changes in MSFP compounding protocols for pf-MPA were noted in the investigation suggesting that sporadic contamination of vials of pf-MPA from MSFP could have occurred in lots without microbiological testing including lots manufactured prior to the start of the investigation period, December 1, 2012. Health care providers should be reminded that if they suspect an adverse reaction from a pharmaceutical product, they should report these reactions to FDA's Medwatch. Although traditional compounding of pharmaceuticals to meet the needs of individual patients has played an important role in treatment, the findings of this investigation contribute to increasing concern about patient safety hazards that can result from the lack of regulatory oversight for large compounding facilities with national product distribution [7-8,10,19,24-26]. To prevent further outbreaks from intrinsically contaminated presumed sterile products, compounding facilities with large distributions should be held to the same standards as other pharmaceutical companies and follow Good Manufacturing Practices [5,7,19,24-26]. Additionally, medical practices need to take proper precautions to ensure injection safety which include recording of lot numbers and developing appropriate written guidelines that follow established standard of care protocols to minimize injection-related adverse events $[2,4,19]$.

\section{Acknowledgment}

All authors report no conflicts of interest relevant to this article. The authors thank everyone at the Florida Department of Health in Washington County, especially Susan Miller, Susie Sewell and Rick Davis for their assistance in this investigation. Thank you to Dr. Tom Török for all his assistance in this investigation. Thank you to the Administrator at the participating medical facility, whose assistance was vital to collecting the necessary information to conduct this investigation. Thank you to the CDC Infectious Diseases Pathology Branch for further examination of samples. Finally, thank you to the Tennessee Department of Health, Tennessee Board of Pharmacy and the Food and Drug Administration for their assistance in this investigation and testing of pharmaceutical samples.

\section{References}

1. Olson RK, Voorhees RE, Eitzen HE, Rolka H, Sewell CM, et al. (1999) Cluster of post injection abscesses related to corticosteroid injections and use of benzalkonium chloride. West J Med 170(3): 143-147.

2. Hutin Y, Hauri A, Chiarello L (2003) Best infections control practices for intradermal, subcutaneous, and intramuscular needle injections. Bull World Health Organ 81(7): 491-500.

3. Villanueva A, Claderon RV, Vargas BA (1997) Report on an outbreak of post injection abscesses due to Mycobacterium abscessus, including management with surgery and clarithromycin therapy and comparison of strains by random amplified polymorphic DNA polymerase chain reaction. Clin Infect Dis 24(6): 1147-1153.

4. Kirschke DL, Jones TF, Stratton CW, Barnett JA, Schaffner W, et al. (2003) Outbreak of joint and soft-tissue infections associated with injections from a multiple-dose medication vial. Clin Infect Dis 36(11): 1369-1373.

5. Galil K, Miller L, Yakrus M (1999) Abscesses due to Mycobacterium abscessus linked to injection of unapproved alternative medication. 
Emerg Infect Dis 5(5): 681-687.

6. http://www.cdc.gov/hai/outbreaks/meningitis-map-large.html

7. http://www.fda.gov/NewsEvents/Testimony/ucm327667.htm

8. Kainer MA, Reagan DR, Nguyen DB, et al. (2012) Fungal infections associated with contaminated methylprednisolone in Tennessee. $\mathrm{N}$ Eng J Med 367(23): 2194-2203.

9. Smith RM, Schaefer MK, Kainer MA (2013) Fungal infections associated with contaminated methylprednisolone injections. N Engl J Med 369(17): 1598-1609.

10. Staes C, Jacobs J, Mayer J, Allen J (2013) Description of outbreaks of health-care-associated infections related to compounding pharmacies, 2000-2012. Am J Health-Syst Pharm 70(15): 1301-1312.

11. http://www.fda.gov/Drugs/DrugSafety/ucm355575.htm

12. http://www.cdc.gov/hai/outbreaks/TN-pharmacy/

13. Haramati N, Lorans R, Lutwin M, Kaleya R (1994) Injection Granulomas intramuscle or intrafat? Arch Fam Med 3(2): 146-148.

14. Reihsaus E, Waldbaur H, Seeling W (2000) Spinal epidural abscess: a meta-analysis of 915 patients. Neurosurg Rev 23(4): 175-204.

15. Tang HJ, Lin HJ, Liu YC, Li CM (2002) Spinal epidural abscess-experience with 46 patients and evaluation of prognostic factors. J Infect 45(2): 76 81.

16. http://labeling.pfizer.com/ShowLabeling.aspx?id=551
17. Bousquet J, Khaltaev N, Cruz AA (2008) Allergic Rhinitis and its Impact on Asthma (ARIA) 2008*. Allergy 63(s86): 8-160.

18. Plaut M, Valentine MD (2005) Allergic Rhinitis. N Engl J Med 353: 19341944.

19. Outterson K. (2012) Regulating compounding pharmacies after NECC. N Engl J Med 367(21): 1969-1972.

20. Michaels L, Poole RW (1970) Injection granuloma of the buttock. Can Med Assoc J 102(6): 626-628.

21. Nisbet AC (2006) Intramuscular gluteal injections in the increasingly obese population: retrospective study. BMJ 332(7542): 637-638.

22. Zaybak A, Gunes UY, Tamsel S, Khorshid L, Eser I (2007) Does obesity prevent the needle from reaching the muscle in intramuscular injections? J Adv Nurs 58(6): 552-556.

23.http://www.fda.gov/downloads/AboutFDA/CentersOffices/ Office ofGlobalRegulatory Operationsand Policy/ORA/ ORAElectronicReadingRoom/UCM356363.pdf

24. Alcorn T (2012) Meningitis outbreak reveals gaps in US drug regulation. Lancet 380(9853): 1543-1544.

25. Savage P (2012) Ensuring the Safety of Compounded Products: Best Clinician Practices and an Existing Solution. Integrative Medicine 11: $17-20$.

26. Drazen JM, Curfman GD, Baden LR, Morrissey S (2012) Compounding Errors. N Engl J Med 367(25): 2436-2437. 